\title{
Application of Reputation Trust Model in C2C E-commerce
}

\author{
Alejandra Lazcano \\ University of Santiago of Chile, Santiago, Chile \\ alejandra.lazcano@usach.cl
}

\begin{abstract}
According to the current status of Consumer-to-Consumer $(C 2 C)$ e-commerce, analyze the differences of factors that affect the evaluation, use the two mechanisms of the time window and transaction window mechanism for the first time to select the evaluation participating in the calculation, and comprehensively consider the self-trust of the evaluator to further improve the evaluation $\mathrm{C} 2 \mathrm{C}$ e-commerce trust evaluation model based on reputation is proposed. This article uses the buyer's understanding ability to assist decision-making, establishes observation groups and other methods to further strengthen the effectiveness of the trust mechanism. The simulation experiment is used to compare the model with the current model. The experimental results show that this model can more truly reflect the authenticity of the evaluation and can be effectively applied to the C2C e-commerce system.
\end{abstract}

Keywords: C2C, Time window, Transaction window, Trust mechanism

\section{Introduction}

With the rapid development of the Internet, e-commerce has developed into a highly influential business model. From eBay and Amazon in the United States to Taobao and JD.com in China, e-commerce has become a must in modern people's lives. The element of preparation. Electronic Commerce (EC) refers to the open network environment of the Internet, based on browsers and other application methods, buyers and sellers carry out various business activities without meeting each other and realizing online transactions and online electronic payments between merchants. A new type of business operation model for various transactions. According to the different participants, it can be divided into the B2B model (Business-toBusiness), B2C model (Business-to-Consumer), and C2C model (Consumer-to-Consumer) [1]. Recently, the C2B model (Consumer-to-Consumer) -Business) also began to rise. E-commerce platforms provide online trading platforms for both buyers and sellers. However, both parties to the transaction, especially in the $\mathrm{C} 2 \mathrm{C}$ model, have to some extent concealed their true information: as well as ignorance of the other party, this will lead to There are great risks in transactions [2][3], such as fraudulent transactions, false information, and the inability to guarantee quality and after-sales service. The security problem in e-commerce is due to the lack of trust, and it seriously affects the development of modern e-commerce. Therefore, how to establish an effective trust evaluation mechanism is to solve a core problem of current ecommerce.

At present, scholars at home and abroad have proposed a variety of trust evaluation models in e-commerce to reduce the occurrence of malicious behaviors in e-commerce. Among them, the use of a reputation mechanism is a typical trust evaluation method. Its basic idea is to allow

Article history:

Received (August 1, 2013), Review Result (October 2, 2013), Accepted (November 5, 2013) 
the parties involved in the transaction to score each other after the transaction is over, which can be divided into positive (Positive, 1 point), neutral (Neutral, 0 points), and negative (Negative, -1 point). The system then scores each person and calculates the individual's trust value. In this way, the user can evaluate the counterparty during the transaction based on the information provided by the system to decide whether to conduct a transaction with the counterparty. This mechanism encourages transaction participants to obtain praise from other participants to obtain a higher reputation. The feedback system in the famous foreign ecommerce website eBay [4][5] adopts this mechanism and is widely used. However, this method sometimes cannot distinguish whether the feedback is real or maliciously slander [6]. For this reason, it is necessary to further determine the authenticity and credibility of the evaluation. Aberer et al. [7] put forward the time factor and transaction amount in its trust model as the weighting factor of trust calculation to improve the credibility of reputation value calculation. Louisot [8] proposed local reputation and global reputation to improve the accuracy of reputation calculation and used a quality model to ensure the authenticity of the evaluation of transaction participants. Mirvis et al. [9] further improved various factors affecting evaluation, including the credit reputation of transaction participants, transaction amount, transaction time, and the experience of both parties to the transaction. From these aspects, the evaluation weight and attenuation were calculated, and dynamics were adopted. The time window to monitor the changes of transaction participants in real-time, fully integrate the factors that affect the evaluation weight and make the evaluation system more comprehensive. Shi et al. [10] introduces cloud model theory into the trust evaluation model of e-commerce so that the model can deal with the very complicated and random environment in e-commerce.

In addition, scholars at home and abroad have also proposed a variety of trust evaluation models in e-commerce, such as the introduction of fuzzy sets of trust models [11][12], the separation of roles of trust evaluation models, etc. [13][14][15], although trust from other perspectives The model has been greatly improved and developed, but it lacks consideration of factors affecting evaluation in credit feedback, ignores the actual situation in today's ecommerce, and does not consider the differences in the impact of various factors on evaluation, making the evaluation true The lack of reliability will eventually cause the evaluation results to deviate from the real situation.

This article conducts an in-depth investigation of the current status of $\mathrm{C} 2 \mathrm{C}$ e-commerce, analyzes the differences in factors that affect evaluation, and combines the current status of ecommerce. The two mechanisms of the time window and transaction window are used for the first time to select the evaluation participating in the calculation and comprehensively consider the evaluator. To further improve the credibility of the evaluation, the reputation-based $\mathrm{C} 2 \mathrm{C} \mathrm{e}$ commerce trust evaluation model is finally proposed. In addition, using the buyer's understanding ability, the model provides decision-making assistance for buyers when choosing a transaction party, to further protect the rights of buyers and increase the success rate of transactions; establish an observation group to supervise them. Finally, through experimental verification and data analysis, this model has good correctness and practicability and can be effectively applied to $\mathrm{C} 2 \mathrm{C} \mathrm{e}$-commerce systems.

\section{Research content}

This article uses the definitions of trust and reputation given by Gambetta [16] and AbdulRahman and Haile [17]: 
Trust (Trust, T): The trust of individual A to individual B is the subjective possibility that individual A expects individual B to serve A (that is, perform actions on which A's interests depend).

Reputation (R): Reputation is the expectation of the individual's future behavior based on the observation or evaluation information of an individual's historical behavior. In e-commerce, it is the trust value of the seller among the transaction participants.

Direct Trust (Direct Trust, DT): In e-commerce, the first-hand in direct transactions with sellers, based on the direct experience of the interaction.

Recommendation Trust (Recommend Trust, RT): In e-commerce, the boss obtains the seller's recommendation trust value, that is, the seller's reputation, through the evaluation value of other bosses on the seller.

Comprehensive trust value (T): In e-commerce, it is a function of the direct trust of the leader and the recommended trust value of the seller. The calculation formula is as formula (1):

$$
T=\alpha D T+\beta R T, \alpha, \beta \in[0,1]
$$

Where $\alpha$ and $\beta$ are confidence factors and their specific calculations will be given later, and they will determine the weights of direct trust and recommended trust.

\section{Trust evaluation model}

\subsection{Analysis of factors affecting evaluation}

In the current trust mechanism, the factors that affect evaluation are mainly considered as transaction time, transaction amount, and the attributes of the evaluator (such as creditworthiness). However, in the development process of e-commerce, to solve the shortcomings, it has also made continuous progress and improvements. For example, Alipay is waiting for the support of such payment software, so it has also changed the role of various influencing factors in the process of trust evaluation. Therefore, it is necessary to further improve and perfect the evaluation system.

In this article, first, analyze the impact factors of these impact evaluations, and extract the most suitable factors. At present, the scale of e-commerce continues to expand, attracting many self-employed operators and consumers, and the transaction volume is constantly breaking new records. This has also led to different scales of operators and different consumer groups.

Transaction time, this evaluation factor refers to the time difference between the transaction occurrence time and the calculation of the credit value. The longer the evaluation distance, the lower the evaluation weight. Then, in today's e-commerce, a time window mechanism is generally adopted, and the displayed evaluation content is the average value of the evaluation within half a year. However, due to the huge difference in the number of transactions of various sellers in e-commerce websites, some sellers have more than hundreds of thousands of evaluations within half a year, while some sellers have only a few hundred or even fewer evaluations. With such a huge gap, if the attenuation of transaction time is adopted, the reputation value of sellers with small transaction volumes will be lower. However, if only such a large time window is adopted without attenuation, it will cause sellers with larger transaction volumes to lose money. After accumulating a large number of buyer reviews, new reviews will be difficult to reflect. In this article, two sets of mechanisms are used for control, time window attenuation processing between machines, which is considered to be authentic data shortly, and sellers with a large transaction volume is segmented according to the transaction window within the time window to obtain Its credit fluctuation graph for better results. 
Transaction amount, the existence of this evaluation factor, is to strengthen the weight of large-value transactions and prevent sellers from using small-value transactions to accumulate credit in transactions, and fraud in large-value transactions. However, in current e-commerce, cash transactions are no longer directly carried out by buyers and sellers, and diversified payment prevention such as cash on delivery and Alipay can effectively prevent fraud in cash transactions. Therefore, in this model, the influence of transaction amount is not considered, and each transaction is considered to have the same evaluation weight.

The attribute of the evaluator generally refers to the credit/reputation value of the evaluator. When evaluating sellers, it is also very important to consider the credibility of the evaluator's evaluation and whether there is malicious slander. Buyers are also gaining a higher degree of credit in continuous transactions, which can increase the weight of their evaluations. In addition, the buyer's ability to understand another attribute of the buyer is also very important. This is only when a certain amount of online shopping experience is needed to continuously enhance it. Therefore, to classify products, build the shopping vector and sales vector VB and VSVB of buyers and sellers to record the number of purchases of buyers and the purchase experience of each category of goods. The purchase experience will be reflected in the selection of sellers. The role of.

Finally, in combination with the current model, it prevents buyers and sellers from using a large number of false transactions and evaluation information to brush high credit with each other. The transaction evaluation of the buyer only takes at most 3 times, and the final evaluation value is calculated; the buyer's multiple evaluations of the same product only take one of the evaluation calculations; the buyer's reputation value growth rate within a certain period is limited, and only the buyer's credit is calculated for the first 5 transactions during this period. In addition, to further strengthen the credibility of the evaluation, for the transaction of non-virtual goods, the seller needs to provide real and queryable logistics information, otherwise, the mutual evaluation information of this transaction will not participate in the credit calculation of the buyer and the seller.

\subsection{Calculation of credit and reputation value}

In calculating the credit and reputation value of the seller and the seller, the weighted average method is used for calculation. To make the evaluation worthy of accuracy, the three values $\{-$ $1,0,1\}$ are not used for credit evaluation, but a 5-point system like [0,5] is used. In the beginning, the seller and the seller did not have any transaction records, and the system will give a lower initial credit value $C_{0}$ and $R_{0}$, which can ensure that the first user who initially entered the system has a low evaluation weight, and further accumulation is required. Get a higher evaluation weight. After the head $i$ has performed $m$ transactions, his credit accumulation is as follows:

$$
C_{i}=\frac{1}{m+1} \sum_{k=0}^{m} c_{k}, c_{k} \in[0,5]
$$

Where $c_{k}$ represents the evaluation made by the seller based on Fengjia's behavior in the $k$ th transaction.

At the end of each transaction, the first family will evaluate the seller's behavior in the transaction. Commonly used evaluation indicators include the degree of conformity between the product and the description, the delivery speed and the service attitude, etc., according to the transaction process. Based on the direct experience of, give the transaction satisfaction $S$ of this transaction, and finally get the evaluation $D T$ of this transaction. Among them, the k-th 
transaction satisfaction $S_{k} \in[0,5]$, the comprehensive evaluation $D T$ is calculated as formula (3):

$$
D T_{k}=\left\{\begin{array}{l}
5 \frac{S_{k}}{R_{k}}, S_{k}<R_{k} \\
5, S_{k} \geqslant R_{k}
\end{array}\right.
$$

If the transaction satisfaction $S_{k}$ is lower than the seller's reputation value $R_{k}$, the transaction satisfaction ratio is used as the evaluation of the seller's reputation; if the transaction satisfaction is higher than the seller's reputation value, it means that the buyer is in the transaction If you are satisfied, you will be given full marks.

After the buyer's evaluation of the seller is generated, the seller's reputation value will be updated accordingly, but according to the different buyer's credit, the weight of the evaluation provided is also different, so seller $\mathrm{j}$ is in the time window or transaction After the nth transaction in the window, the reputation value is calculated as formula (4):

$$
R_{j}=\frac{1}{n+m}\left(\sum_{k=1}^{n} \frac{C_{k}}{5} \cdot D T_{k}+m R_{0}^{\prime}\right)
$$

Where $C_{k}$ represents the credit value of the corresponding leader in the kth transaction, $D T_{k}$ represents the direct evaluation of the corresponding leader to the seller $j$ in the kth transaction, and $R_{0}^{\prime}$ represents the initial credit value, that is, in the last time window or transaction window The accumulated reputation value, $m$ represents the number of retained evaluations. Since it is the reputation accumulated by the transaction, its weight is 1 , and initially $R_{0}^{\prime}=R_{0}$.

\subsection{Other auxiliary strategies}

(1) Decision-making assistance

After the head family has a certain trading experience, the system will classify and count the categories of its head products and calculate the comprehension ability (Savvy) value for it based on the statistical information, which is recorded as Sy. When the head family chooses the transaction party later. According to the value of its understanding ability, the comprehensive trust value to the transaction party is calculated. The comprehension ability of the first user is manifested in two aspects: whether he has transaction experience with the other party and the first user's familiarity with the category of the seller. For this reason, the number of direct transactions is recorded as DTT (Direct Trade Time), which is the transaction vector VB of each first user, which records the number of times the first user for each type of commodity; and the vector VS is established relative to the seller user, and the category belongs to Marked as 1 , others are 0 . The familiarity of the first family $i$ to the seller $\mathrm{j}$ is $\mathrm{F}$, and the calculation formula is as follows (5):

$$
F_{i j}=V B_{i} \cdot V S_{j}
$$

According to whether the first family $i$ has transaction experience with seller $j$ and the first family's familiarity with the seller's category, calculate the first family's ability to understand the sellerSy, as in formula (6):

$$
S y_{i j}=\left\{\begin{array}{l}
\alpha_{1}, D T T_{i j} \geqslant c_{1} \text { 或 } F_{i j} \geqslant c_{2} \\
\alpha_{2}, 0 \leqslant D T T_{i j}<c_{1} H .0 \leqslant F_{i j}<c_{2} \\
0, D T T_{i j}=0 \text { 且 } F_{i j}=0
\end{array}\right.
$$


Where $0<\alpha_{2}<\alpha_{1} \leqslant 1$, where $c_{1}$ and $c_{2}$ are control parameters.

According to the understanding ability $S y$ of the first family $i$, when choosing the seller $\mathrm{j}$ to trade with, the final trust degree $T_{i j}$ can be obtained, and the calculation formula is as (7):

$$
T_{i j}=S y_{i j} \cdot \frac{1}{n+1} \sum_{k=0}^{n} D T_{k}+\left(1-S y_{i j}\right) R_{j}
$$

Where the confidence factor $\alpha=S y_{i j}, \beta=1-S y_{i j}$, when $S y_{i j}>0$ and $D T T_{i j}=0, D T_{k}$ takes the credit of the middle seller in the transaction with this type of buyer, which is closest to the seller's reputation value, and fluctuates up and down as $\pm 0.1, n$ is the number of historical evaluations taken.

(2) Establish an observation group

To further effectively supervise the parties involved in the transaction, an Observation Group (OG) is established to focus on the poorly performing individuals in the system. It is necessary to establish separate observation groups for buyers and sellers. After honest transactions, the transaction participants in the observation group will be removed from the observation group and returned to the normal user group after they reach the normal reputation/credit value; During the observation group, if relevant unfavorable behavior occurs again, the punishment will be increased.

In today's e-commerce, the buyer's credit value is considered very little, but the buyer does have some problems that need to be paid attention to, such as whether to fill in the evaluation information truthfully, and whether to return and exchange frequently. In the process of purchasing goods, buyers refer to the opinions of other consumers as references, and this reference information is a valuable resource for e-commerce to survive. When they enjoy such rights When you have a transaction, you should give true and credible evaluation information for other people to refer to, to fulfill your obligations. If you do not evaluate for a long time, this article believes that this is a behavior that damages its credit value. Its credit value will be deducted. Therefore, for buyers who have repeatedly exhibited the above behaviors, join the observation group $O G_{B}$ and remind users to correct their behaviors.

For sellers, the system groups sellers in the same category according to their classification vector VS. For sellers with poor performance in the same industry, such as low reputation value or high complaint rate, they will be added to the observation group in $O G_{S}$, focus on it.

\section{Simulation experiment and analysis}

This paper simulates the current operating mode of $\mathrm{C} 2 \mathrm{C}$ e-commerce, conducts simulation experiments, and compares it with the current evaluation system. In the experiment, use $\mathrm{C}$ to write the trading platform to simulate the transaction participants in the real $\mathrm{C} 2 \mathrm{C} \mathrm{e}$-commerce: buyer user group and seller user group, randomly select the transaction object, and evaluate each other after the transaction is over, and record the transaction in the database Time, mutual evaluation, etc., and update the changes in the credit value and reputation value of both buyers and sellers in real-time; to further conform to the situation in the real society, set a certain $20 \%$ of buyers and sellers to have a large number of users' transactions, and conduct multiple experiments Calculate the evaluation results of the model proposed in this paper and the current model separately to achieve the authenticity and representativeness of the data. In the experiment, CM (Current Model) is used to represent the evaluation models currently used in major e-commerce platforms, and their evaluation indicators are also compared on a 5-point 
scale; RBM (Reputation Based Model) is used to represent the model in this article. The relevant parameters in the simulation experiment are shown in [Table 1].

Table 1. Parameter settings in the simulation experiment

\begin{tabular}{|c|c|}
\hline Describe & Defaults \\
\hline Number of buyers & 100 \\
\hline Number of sellers & 50 \\
\hline The favorable rate in the evaluation $(4,5)$ & $95 \%$ \\
\hline Percentage of sellers with high transaction volume & $20 \%$ \\
\hline Total number of transactions & 20000 \\
\hline
\end{tabular}

[Figure 1] reflects the performance of two different buyer users in the transaction process. The seller user evaluates them and obtains their credit value during the transaction behavior, which can make the buyer user who just entered the system weigh Lower, the credit value of buyers with more good trading behaviors gradually increases to increase the weight of their evaluation. However, the current methods in major e-commerce platforms, the more the number of transactions, the higher the credit value of the program, this method can better reflect fairness, so that buyers also need to restrict their behavior, not malicious. The credit value of the behavior will continue to increase, otherwise, it will be recognized and punished by the system, which provides a more important foundation for the sound development of the entire system.

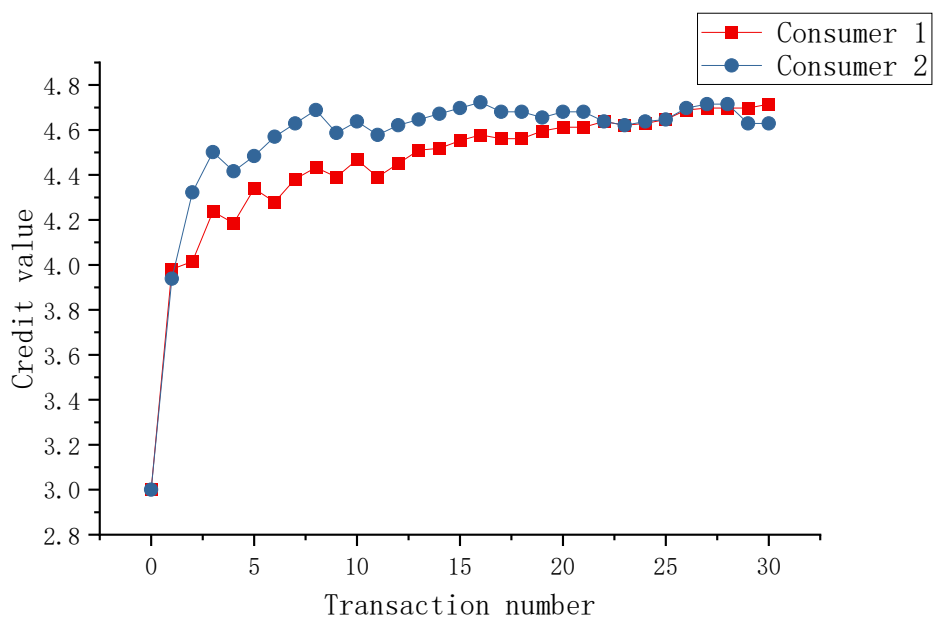

Figure 1. Buyer's credit accumulation

[Figure 2] reflects a seller with a small transaction volume (the transaction window size in this article is set to 200, and when the number of transactions in a time window is not greater than 200, the transaction volume is considered to be less), in a time window, continue to accumulate credit the process of. It can be seen that when buyer credit is added as a weight, the speed of credit accumulation is lower than the accumulation speed in the current model, which can effectively prevent some illegal sellers from using improper means to quickly Raise their credit value to seek illegitimate benefits. 


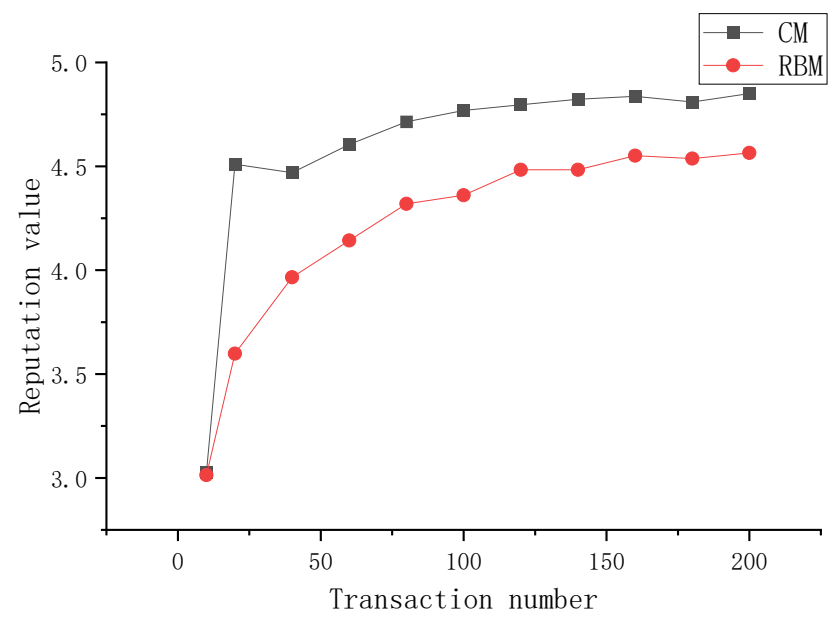

Figure 2. Reputation accumulation of sellers with low transaction volume in a time window

Figure 3 reflects that when the transaction volume of some sellers is large in a time window, the accumulated historical data will cause the new evaluation to not be reflected in the seller's reputation value in a timely and effective manner. Using the current calculation Method, the change of the curve tends to be flat, which is not conducive to reflecting the seller's true reputation, and as the transaction volume continues to increase, the effect will become less obvious, so that the reference meaning for buyers will become less and less. For the same seller, when the transaction volume in a time window increases, the model in this paper uses the transaction window to further refine the change process of its reputation value, to correctly reflect the small-time periods in the transaction process (Corresponding to the transaction period within a transaction window) credit change. As shown in [Figure 4], it reflects the change in the reputation of the seller in the fifth transaction window of the transaction. It can be seen that while the transaction volume is accumulating, the existing model has almost lost its due reference value, and the model in this paper can still reflect the changes in the seller's reputation value, with higher real-time and accuracy. Can give consumers a true and effective reference standard.

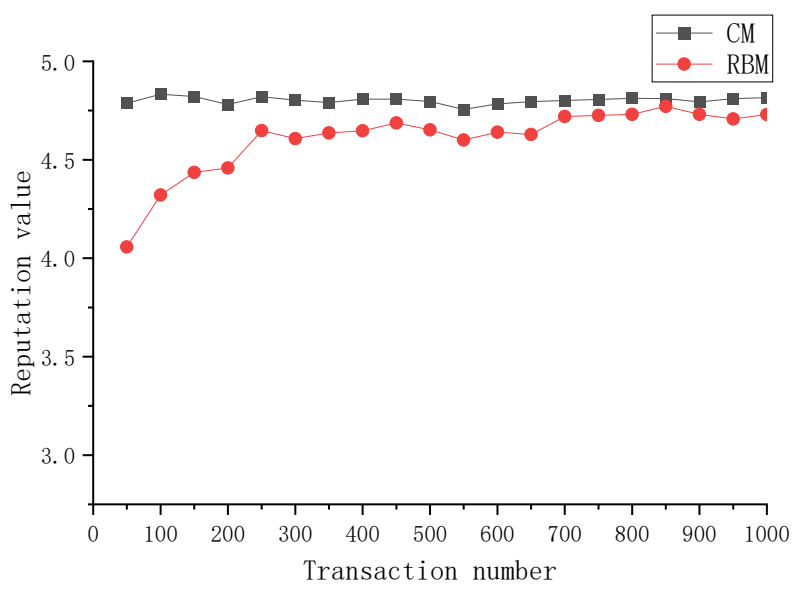

Figure 3. Reputation accumulation of sellers with multiple transactions within a time window 


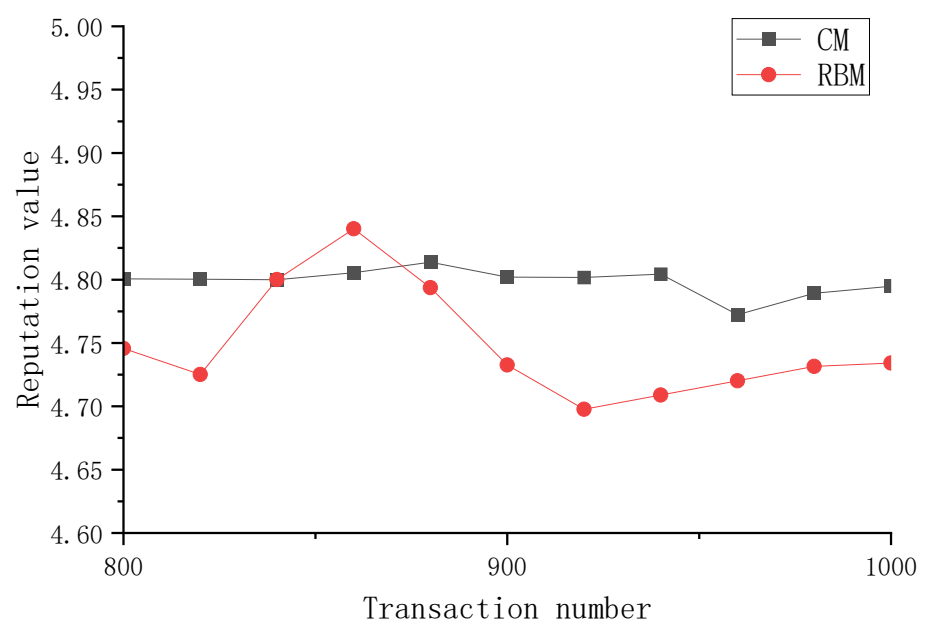

Figure 4. The seller's reputation changed in the last transaction window

In [Figure 5], situation 1 reflects that the buyer has not had a transaction with the seller, $S y_{i j}=0$, and is not familiar with the goods sold by the seller, and cannot provide reference information; situation 2 reflects that the buyer has certain Purchase experience, and have historical transactions with the seller, $S y_{i j}=\alpha_{2}$ but the seller's evaluation is not high, so the system reduces the seller's reputation for this buyer; Scenario 3 reflects that the buyer has a certain degree Purchase experience and high evaluation of the seller's historical transactions (fewer times), $S y_{i j}=\alpha_{2}$, so the system improves the seller's reputation value; Scenario 4 reflects that the buyer and the seller have conducted more transactions, or It has a lot of buying experience. The $S y_{i j}=\alpha_{1}$ system, based on its historical data, greatly improves the buyer's reputation for this seller. Through four different situations, different buyers make their judgments on the same seller, which can better help users decide whether to trade with the seller, which can bring greater convenience to consumers.

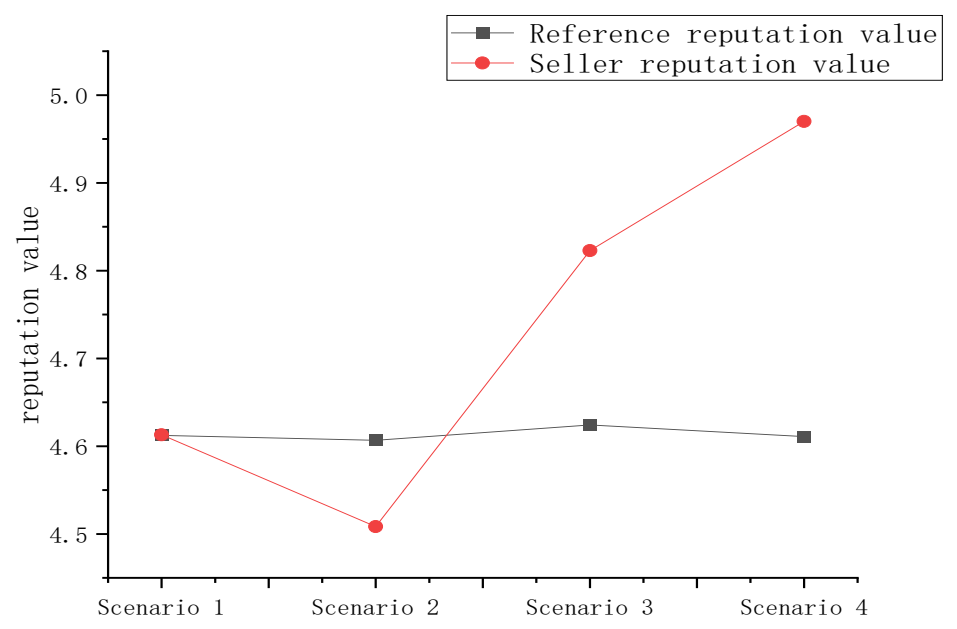

Figure 5. The reference of the auxiliary decision-making system to buyers 


\section{Conclusion}

Given the current problems in $\mathrm{C} 2 \mathrm{C}$ e-commerce, after further research and analysis of factors that affect the evaluation, combined with the current third-party secure payment methods widely used in e-commerce, this paper proposes a reputation-based $\mathrm{C} 2 \mathrm{C}$ e-commerce trust evaluation model, using time windows and the transaction window mechanism selects the evaluation participating in the calculation, and comprehensively considers the self-trust of the evaluator. And use the buyer's understanding ability to assist decision-making, establish observation groups, and other methods to further strengthen the effectiveness of the trust mechanism. Finally, a simulation experiment is used to compare the model with the current model. The experimental results show that this model can more truly reflect the authenticity of the evaluation and can be effectively applied to the $\mathrm{C} 2 \mathrm{C}$ e-commerce system.

\section{References}

[1] K. Jones and L. Leonard, "Trust in consumer-to-consumer electronic commerce," Information and Management, vol.45, no.2, pp.88-95, (2008)

[2] E. N. Billingsley, L. Monier, and A. Arora, "Method and system to facilitate a search of an information resource," (2012)

[3] A. Alghamdi, H. Aldabbas, and M. Alshehri, "Adopting user-centered development for Arabic e-commerce websites," International Journal of Web and Semantic Technology, (2012)

[4] L. Jian, J. K. Mackie-Mason, P. I. Resnick, "Scratched yours: The prevalence of reciprocation in feedback provision on eBay," B.E. Journal of Economic Analysis and Policy, vol.10, no.1, (2010)

[5] S. Robert and G. Tapan, "Remain silent and ye shall suffer: seller exploitation of reticent buyers in an experimental reputation system," Experimental Economics, (2011)

[6] S. S. Standifird, "Reputation and e-commerce: eBay auctions and the asymmetrical impact of positive and negative ratings," Journal of Management, vol.27, no.3, pp.279-295, (2001)

[7] K. Aberer and Z. Despotovic, "Managing trust in a peer-2-peer information system," (2001)

[8] J. P. Louisot, "Managing intangible asset risks: Reputation and strategic redeployment planning," Risk Management, vol.6, no.3, pp.35-50, (2004)

[9] Mirvis and H. Philip, "Stakeholder engagement: Local faces and global reputation," Corporate Citizen Magazine, no.4, pp.9-22, (2010)

[10] Y. Z. Shi and H. C. Zhou, "Research on monthly flow uncertain reasoning model based on cloud theory," Science China, vol.53, no.009, pp.2408-2413, (2010)

[11] Keon, Myung, Lee, "A fuzzy trust model incorporating dispositional trust, general trust, situational trust and reputation," Journal of Korean Institute of Intelligent Systems, vol.16, no.6, pp.653-658, (2006)

[12] M. Rehak, L. Foltyn, and M. Pechoucek, "Trust model for open ubiquitous agent systems," // IEEE/WIC/ACM International Conference on Intelligent Agent Technology, ACM, 2005,

[13] N. N. Cheng and B. Yang, "A role-based trust-management model with degrees of trust," Application Research of Computers, (2006)

[14] D. Kovac and D. Trcek, "Qualitative trust modeling in SOA," Journal of Systems Architecture, vol.55, no.4, pp.255-263, (2009)

[15] K. M. Lee, K. S. Hwang, and J. H. Lee, “A fuzzy trust model using multiple evaluation criteria," Springer Berlin Heidelberg, (2006)

[16] D. Gambetta, "Can we trust: Making and breaking cooperative relations, pp.213-237, (2000)

[17] A. Abdul-Rahman and S. Hailes, "Supporting trust in virtual communities," //Proceedings of the 33rd Annual Hawaii International Conference on System Science, vol.1, no.9, (2000) 